\title{
Beam test of scintillation tiles with MPPC readout $\left(^{*}\right)$
}

\author{
A. Calcaterra, R. De Sangro, G. Finocchiaro, P. Patteri, \\ M. PiCCOLO and M. RAMA \\ INFN, Laboratori Nazionali di Frascati - Via E. Fermi 40, I-00044 Frascati (Rome), Italy
}

(ricevuto il 3 Marzo 2008; pubblicato online il 24 Aprile 2008)

\begin{abstract}
Summary. - This paper describes measurements made using counters composed of a small ( 3 by 3 by $0.5 \mathrm{~cm}^{3}$ ) scintillation tile coupled to a Multi-Photon Pixel Counter (MPPC) produced by Hamamatsu and exposed to an electron beam at the Beam Test Facility in Frascati. We show our first results for charge spectra and efficiency, and a very preliminary measurement of device linearity.
\end{abstract}

PACS 29.40.Wk - Solid-state detectors.

PACS 29.40.Mc - Scintillation detectors.

PACS 29.40.Gx - Tracking and position-sensitive detectors.

\section{1. - Introduction and motivation for this study}

Silicon photomultipliers [1], often called "SiPM" in the literature, are semiconductor photon detectors built from a square matrix of avalanche photodiodes (APDs) on common silicon substrate. The dimension of each single APD square microcell can vary from 20 to $100 \mu \mathrm{m}$. Every microcell - or pixel - operates in Geiger mode and is decoupled from the adjacent ones by a polysilicon quenching resistor, and microstructures called "trenches".

Although individual pixels work in digital mode, the SiPM is an analog device because the signal is the sum of all pixels crossed by a photon, and may vary in a range from a single pixel to hundreds of pixels per square millimeter; if the number of photons impacting the device is much smaller than the total number of pixels on the device, and inter-pixel optical crosstalk can be neglected, the signal is proportional to the number of original photons.

Several factories already sell SiPMs, in particular Hamamatsu [2] (called MPPCs) and Photonics [3]. Others, among which the Italian IRST [4], work mainly in collaboration with INFN and aim to further understanding and development of this device.

(*) Paper presented at the 1st Workshop on Photon Detection for High Energy Medical and Space Applications; Perugia, June 13-14, 2007.

(C) Società Italiana di Fisica 


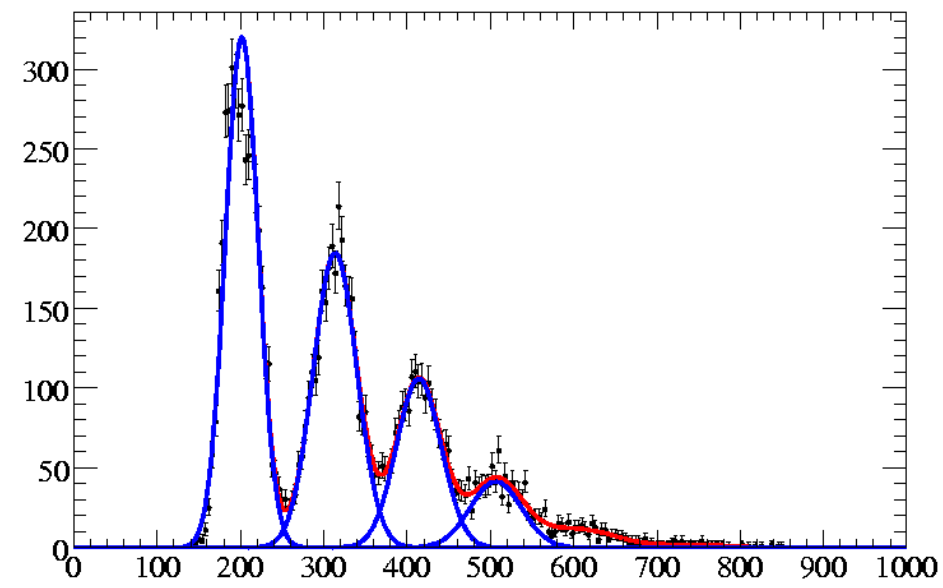

Fig. 1. - Beam pulse multiplicity from the BTF Pb-glass calorimeter. The peak at $\sim 200$ counts corresponds to 0-MIPs beam pulses.

The applications of silicon photon detectors are very wide [5]; this particular study originated in the context of calorimetry for detectors to be installed at the International Linear Collider.

The demands imposed by physics to calorimetric detectors for the ILC are such that the requested energy resolution seems only obtainable using "Particle Flow" reconstruction algorythms, based on the separation between the neutral and charged particle components of a shower, and thus necessitating a very fine granularity and compact packing of individual detection elements; a tile of 30 by 30 by $5 \mathrm{~mm}^{3}$, and possibly thinner, being a typical one.

A real-life prototype [6] has already been developed and exposed to test beams at CERN and FNAL, by the CALICE Collaboration; repeating an effort on such a scale would not be practical. We thus aimed at a study of a few stand-alone counters, exposed to single particles, to obtain properties not easily accessible to the big prototype.

\section{2. - The Beam Test Facility in Frascati}

The Beam Test Facility [7] exploits by means of a transfer line the DA $\Phi$ NE $\phi$-factory LINAC, and is optimized for the production of electron and positron bunches in a wide range of multiplicities, down to a single electron.

The BTF may be run in "dedicated" mode, with the beam energy tunable over an ample range $(50-500 \mathrm{MeV})$ or in "parasitic" mode, using the time slots in which the $\mathrm{DA} \Phi \mathrm{NE}$ complex is not injecting the main storage rings.

The maximum repetition rate is $50 \mathrm{~Hz}$, and the maximum pulse multiplicity depends on the repetition rate: current safety regulations impose a maximum particle flux of $1 \mathrm{kHz}$, so that at $50 \mathrm{~Hz}$ the maximum multiplicity in one pulse is 20 particles. At $1 \mathrm{~Hz}$, the system may deliver pulses containing $\sim 1000$ particles.

The beam profile from the BTF has typical horizontal and vertical dispersions of $\sigma_{h}=2 \mathrm{~mm}$ and $\sigma_{h} \leq 5-10 \mathrm{~mm}$, with some additional, wider halo in the horizontal plane, where the bending magnets act.

The set-up available to users of the BTF includes a Pb-glass calorimeter, having dimensions 10 by $10 \mathrm{~cm}^{2}$ by $\sim 20 X_{0}$, to be placed downstream the user set-up, read by 


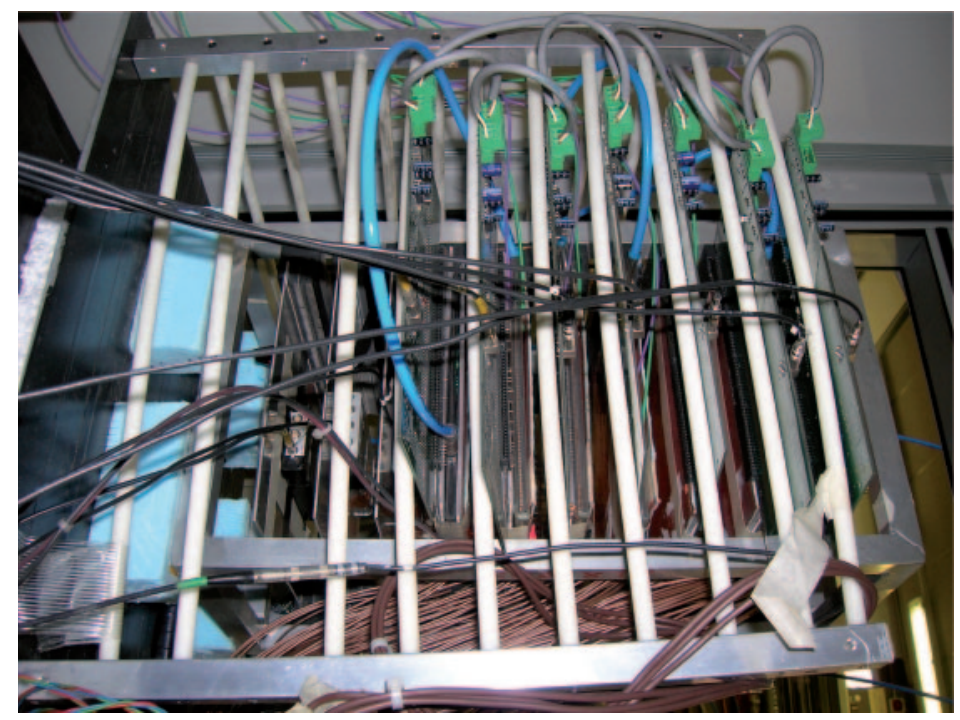

Fig. 2. - A view of the experimental set-up. The beam enters from the right.

a PM gated at every beam pulse. The beam pulse is totally absorbed in the calorimeter, and the integrated signal from the PM gives a measurement of the multiplicity, as shown in fig. 1, where the peaks due to 0-, 1-, 2- and more particle events are clearly separated.

In most of this work, and unless otherwise stated, only events having a Pb-glass calorimeter signal in the second peak have been used: those corresponding to beam pulses of multiplicity exactly one.

\section{3. - Description of the set-up}

The set-up, shown in fig. 2, is composed of 3 MPPC detectors [2] and a 6-layer, twodimensional tracker made of mechanically-quenched Resistive Plate Counters (RPC); the purpose of the tracker is to measure the beam impact point on the scintillation tiles but, as the present analysis does not address the issue of pulse height vs. impact points, its information was not used for this paper.

The set-up is triggered for every beam pulse via a signal synchronous with the LINAC radiofrequency; our trigger is thus absolutely independent of our measurements and the events are completely unbiased. A view of the set-up is given in fig. 2.

3•1. The RPC tracker. - The RPCs used in this test are based on a $2 \mathrm{~mm}$ gas gap and employ resistive glass plates; in the gas flow a honeycomb structure is placed, that realizes a mechanical quenching of the showers and allows the device to be run without freon. More details are given in ref. [8].

On the 2 readout layers of each RPC two orthogonal strip systems with 16 strip each are machined, having pitch of $\sim 7.8 \mathrm{~mm}$. These 2 views allow us to obtain a single-layer resolution at the level of a few $\mathrm{mm}$, as shown in fig. 3 .

The readout for the tracker proceeds via custom electronics and VME modules. 

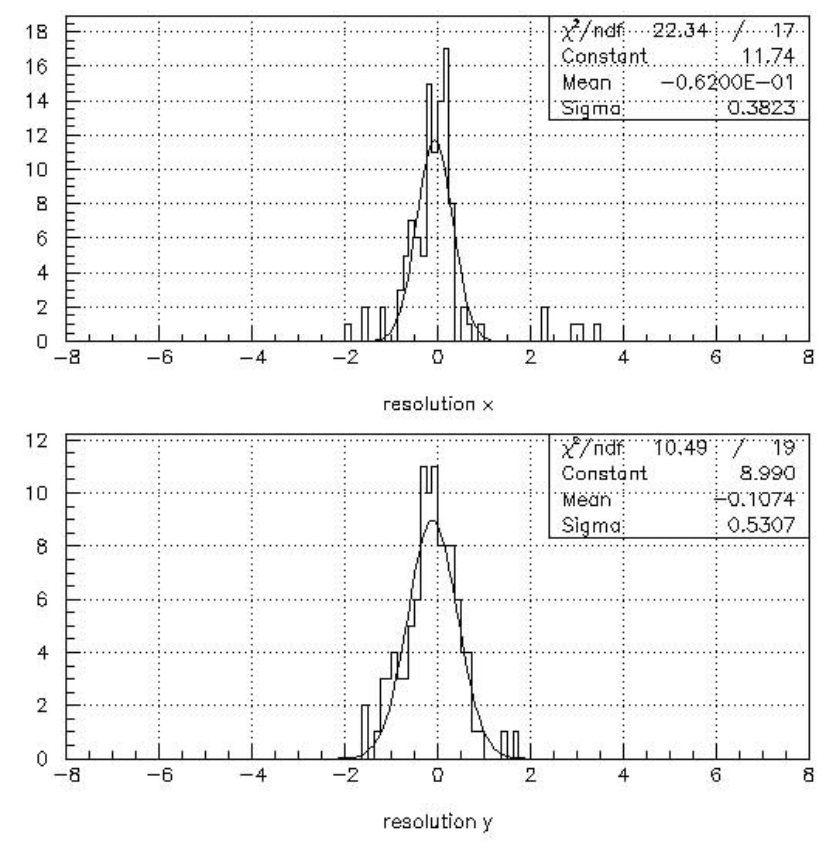

Fig. 3. - Horizontal(up) and vertical fitted resolution typical of the RPC tracker. The point in the layer plotted is excluded from the fit.

3.2. The MPPC detectors. - The counters used in this set of measurements were 3 , differing only for the scintillating material and the MPPC type. One (opened) assembly appears in fig. 4, where one can see a cut breadboard inside a sturdy, light-tight $\mathrm{Al}$ box; inserted in the breadboard is the tile (covered with black tape); mounted on the breadboard is a preamplifier from Photonique [3] with a gain $\sim 15$, connected to a MPPC inserted into the tile side. The MPPC signal exits via the LEMO connector mounted on the box top side, and is integrated on a VME 12-bit ADC (CAEN V792) using a 200 ns gate synchronous with the beam pulses. The "live" part of the boxes was:

- for counter "1", a scintillation tile made of St. Gobain BC-400 (blu-scintillating material, equivalent to Nuclear Enterprises NE-102), coupled to the MPPC using a green fiber of thickness $1 \mathrm{~mm}$, inserted into a groove machined along the tile center. The MPPC had 1600 pixels arranged in a square matrix of 1 by $1 \mathrm{~mm}$, and the pixel pitch was $25 \mu \mathrm{m}$.

- For counter "2", a scintillation tile made of green-scintillating material, similar to Scionix EJ260, coupled to the MPPC analogously to counter "1". The MPPC had 400 pixels arranged in a square matrix of 1 by $1 \mathrm{~mm}$, and the pixel pitch was $50 \mu \mathrm{m}$.

- For counter "3", a scintillation tile like counter "1", directly coupled to the MPPC without a fiber. The MPPC was like the one in counter "2".

In all 3 counters the MPPCs were biased using a power supply HP6614C, with a stated accuracy of $0.03 \%$, in quadrature with $12 \mathrm{mV}$. 


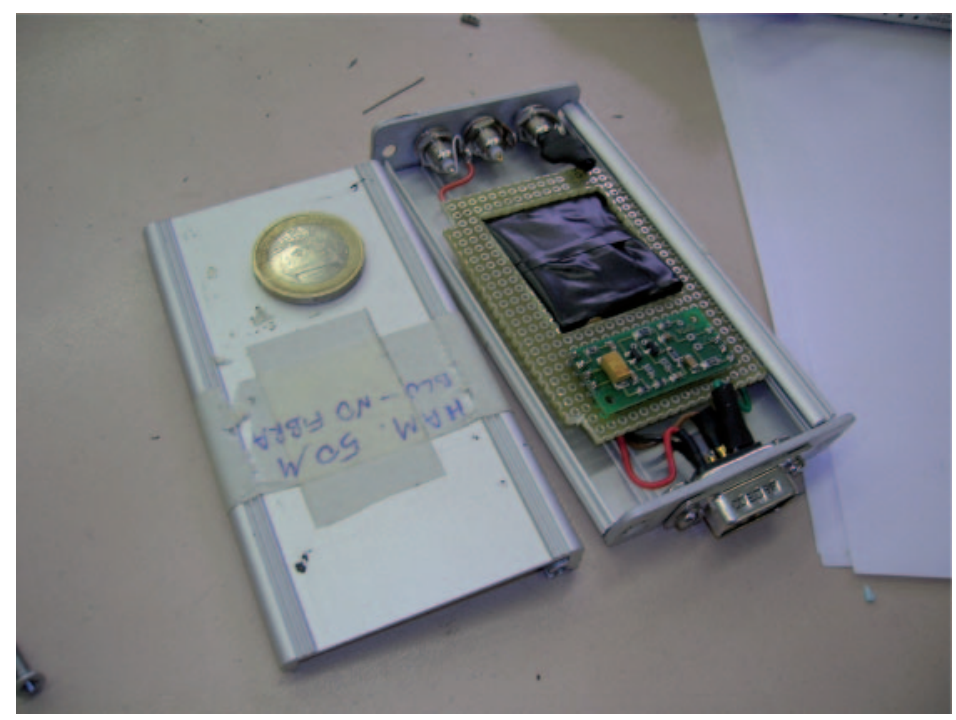

Fig. 4. - A counter assembly, showing the 3 by $3 \mathrm{~cm}^{2}$ tile and the preamplifier.

\section{4. - Measurements}

4.1. Charge spectra and gains. - Figure 5 shows the pedestal-subtracted charge spectra (in pC) for counters 2 (up) and 3 biased at $69.5 \mathrm{~V}$, about $1 \mathrm{~V}$ above the MPPC breakdown voltage; they both have a 400-pixels MPPC, but differ by the type of scintillator, and the type of coupling (see above). Exactly 1 particle per beam pulse has been requested here, using a cut on the Pb-glass calorimeter signal selecting the second peak in fig. 1 . The contribution from events having 2 particles per pulse is estimated at $\sim 5 \%$.
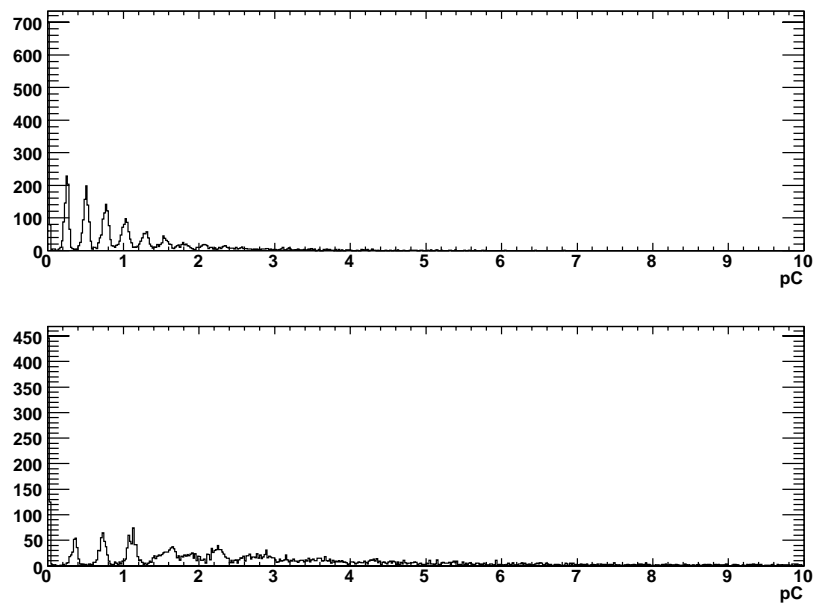

Fig. 5. - Charge spectra in pC for counters $2(\mathrm{up})$ and 3 ( $V_{\text {bias }}=69.5 \mathrm{~V}$ for both). 


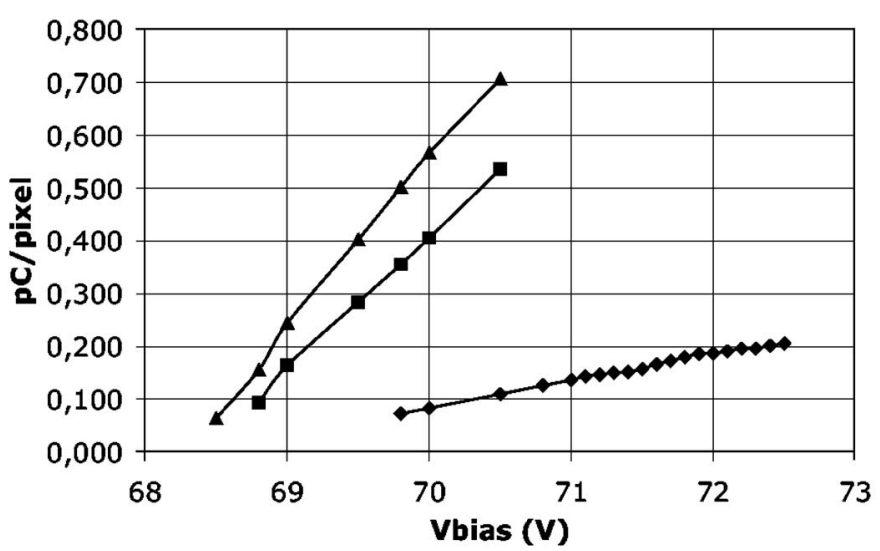

Fig. 6. - Gains for the three counters studied (see text for details).

Both spectra have a very narrow peak at 0 , barely visible in the picture, and a series of equally spaced peaks, due to events firing $1,2,3$, and more pixels in the MPPCs. The RMS noise, estimated by fitting with a Gaussian the peak at $0 \mathrm{pC}$ is $2-3 \mathrm{fC}$ and the gain, measured by fitting the peak pitch, is $\sim 1.6 \times 10^{6}$ for counter 2 and $\sim 2.2 \times 10^{6}$ for counter 3 . In fact, the peaks from counter 3 are visually more widely spaced than those from counter 2 .

Since the preamplifiers for all counters were equal, and all 3 channels of electronics identical, the gain difference indicates that the working points for the 2 MPPCs were actually different: the scintillator material and the coupling geometry would have rather affected the peak populations, creating a bigger or smaller "average number of peaks".

These plots also show signs of deterioration of the charge signal beyond $\sim 0.150 \mathrm{pC}$, not attributable to channel electronics, the RMS noise figure (width of the peak at $0 \mathrm{pC}$ ) being about the same for both.

A measurement of the gain for all 3 MPPCs as a function of the biasing tension $V_{\text {bias }}$ is shown in fig. 6; the data shown are for counter 1 (diamonds), 2 (squares) and 3 (triangles). From the comparison, the 1600-pixels MPPC needs a higher $V_{\text {bias }}$ to obtain

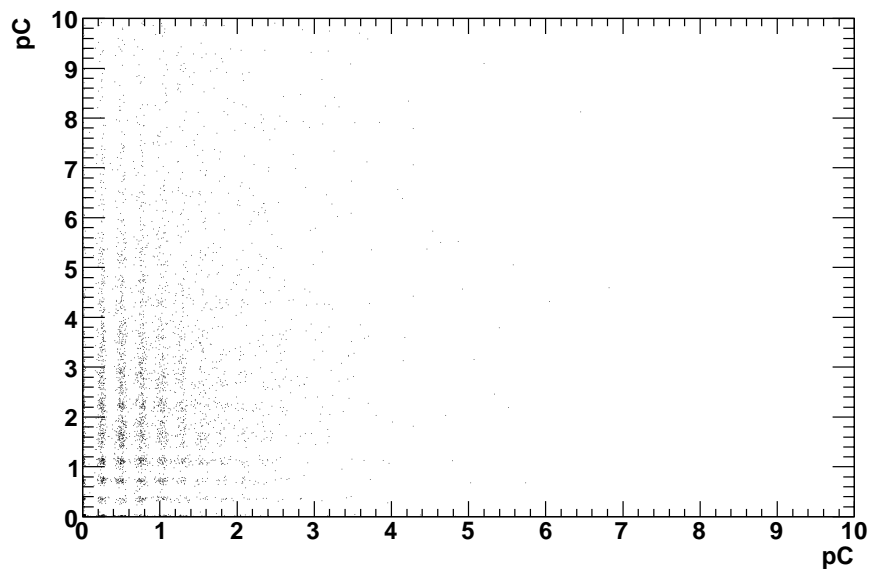

Fig. 7. - Integrated charge from counter 2 (horizontal axis) and 3. 


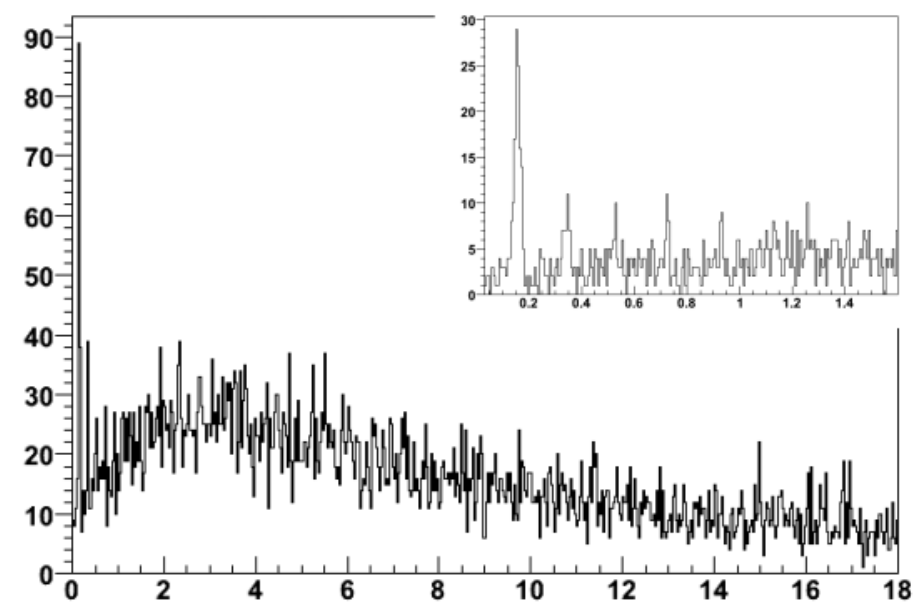

Fig. 8. - Charge spectrum in $\mathrm{pC}$ for counter 1. In the inset, on an expanded scale, the pedestal region.

the same gain as the others, that are moreover not identical. Counter 2 needs a higher $V_{\text {bias }}$ than counter 1 , for the same gain.

4. Efficiency. - We measured the efficiency of counters 2 and 3 by plotting the signal from counter 2 along the horizontal axis of fig. 7 and the signal from counter 3 vertically.

Cutting slightly above the pedestal $(\sim 4 \sigma$ 's) for one of the two, due to the very close proximity of the 2 scintillating tiles, we evaluate the efficiency of the other one as the ratio of above-pedestal signals to the total for the events left by the former cut. These proximity-defined efficiencies are of $(84 \pm 1) \%$ and $(91 \pm 1) \%$ for counters 2 and 3 , respectively. We attribute the difference in efficiencies to the different working point of the 2 MPPCs, that were identically biased at $69.5 \mathrm{~V}$.
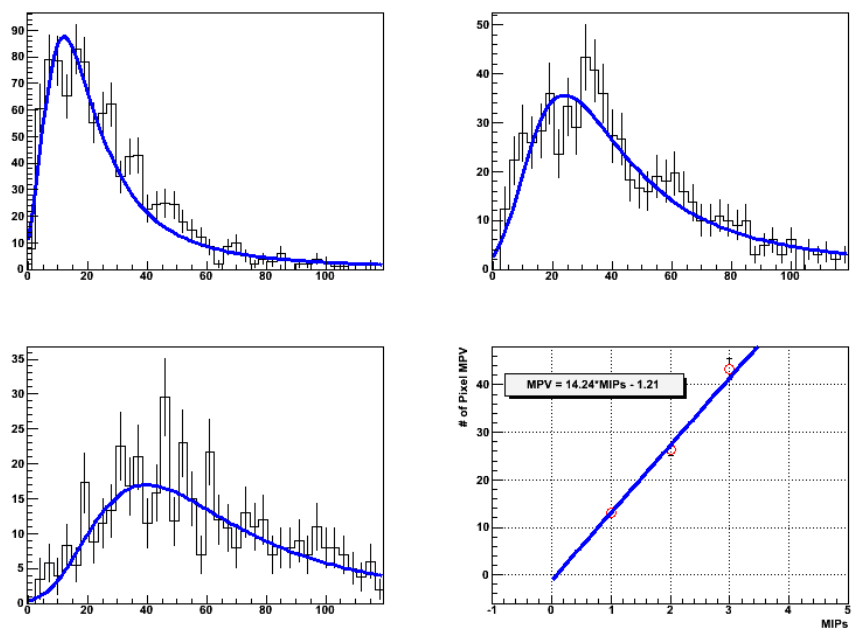

Fig. 9. - Linearity of counter 1 readings. The top and bottom left plots are charge spectra in units of "pixels" and the curves are fits to the Landau distribution. In the bottom right plot we see the MPVs of the Landau distributions plotted against the number of MIPs in the beam pulses. 
4.3. Linearity. - In fig. 8 one may see the charge spectrum in $\mathrm{pC}$ for counter 1 , biased at $72.0 \mathrm{~V}$. At first sight, the pixel structure is less visible than for counters 2 and 3 , but it is still present, as shown in the inset, that displays on an expanded scale the region close to the pedestal.

Dividing the data in fig. 8 by the gain, one gets the histogram shown in the top left part of fig. 9, where the charge axis is now in "pixel" units. Repeating this procedure for events selected in the 2- and 3-particle region, given by the 3rd and 4th peak in fig. 1, one obtains the top right and bottom left plots in fig. 9 .

Fitting the data to Landau distributions we obtain the Most Probable Values (MPVs) plotted in the bottom right part of fig. 9. The fitted line is a measurement of the most probable number of pixels fired in the MPPC of counter 1 as a function of the number of MIPs in the beam pulses. The points lie very close to the line: the fitted number of pixels per MIP is 14, and the extrapolated line passes close to the origin.

\section{5. - Conclusions and outlook}

The results described in this paper, although still in an initial stage, encourage us to proceed with the study of the performance in a beam test and in cosmic rays of scintillation counters employing a SiPM as detection element: with a small and relatively fast assembly we have obtained charge spectra and efficiency, and a first very preliminary measure of linearity.

Using these detectors on a large scale (millions of MPPCs) and obtaining the energy resolution needed for ILC calorimetry requires detailed understanding of SiPM-to-SiPM variations. In this sense, the fact that our very first pair of identical MPPCs presents a difference of $\sim 0.5 \mathrm{~V}$ in the working point may be fortuitous, or may be an indication that the industry needs more accuracy in the fabrication.

We plan to accumulate more statistics increasing the number of counters studied by an order of magnitude, and take more data at the BTF and in cosmic rays, where the small rate may be offset by continuous data taking. We will also implement a system to read the temperature of the devices under test simultaneously with data taking, to measure the variation of MPPCs gain as a function of $T$.

$$
* * *
$$

The authors of this paper wish to express their thanks to G. MAZziTELLI and P. VALENTE, and all operators of the BTF facility for successful and time-efficient running, and to L. DANIELLO for his skill and dedication in the assembling of the MPPC counters.

\section{REFERENCES}

[1] Dolgoshein B. et al., Nucl. Instrum. Methods A, 563 (2006) 368, and references therein.

[2] The present paper is about devices S10362-11-050U and S10362-11-025U, for a description and more types, see http://www.hahamatsu.it.

[3] See the Photonique homepage, http://www.photonique.ch/index.html.

[4] See the contribution by C. Piemonte, this issue, p. 473.

[5] For a recent review, see N. OTTE in Proceedings of the SNIC April 2006 Symposium, SLAC, Stanford, available online at http://www-conf.slac.stanford.edu/snic/default.htm.

[6] Andreev V. et al., Nucl. Instrum. Methods A, 540 (2005) 368.

[7] Mazzitelli G. et al., Nucl. Instrum. Methods A, 515 (2003) 516. See also the web page http://www.lnf.infn.it/acceleratori/btf/publications.html.

[8] Calcaterra A. et al., Nucl. Instrum. Methods A, 565 (2006) 444. 